\title{
Water absorption through salivary gland type I acini in the blacklegged tick, Ixodes scapularis
}

\author{
Donghun Kim $^{1}{ }^{\text {， Paulina Maldonado-Ruiz }}{ }^{1}$, Ludek Zurek ${ }^{1}$, Yoonseong Park ${ }^{\text {Corresp. } 1}$ \\ 1 Department of Entomology, Kansas State University, Manhattan, Kansas, United States \\ Corresponding Author: Yoonseong Park \\ Email address: ypark@ksu.edu
}

Tick salivary glands play critical roles in maintaining water balance for survival, as they eliminate excess water and ions during blood feeding on hosts. In the long duration of fasting in the off-host period, ticks secrete hygroscopic saliva into the mouth cavity to uptake atmospheric water vapor. Type I acini of tick salivary glands are speculated to be involved in secretion of hygroscopic saliva based on ultrastructure studies. However, we recently proposed that type I acini play a role in resorption of water/ions from the primary saliva produced by other salivary acini (i.e., types II and III) during the tick blood feeding phase. In this study, we tested the function of type I acini in unfed female Ixodes scapularis. The route of ingested water was tracked after forced feeding of water with fluorescent dye rhodamine123. We found that type-I acini of the salivary glands, but not type II and III, are responsible for water uptake. In addition, the ingestion of water through the midgut was also observed. Injection or feeding of ouabain, a $\mathrm{Na} / \mathrm{K}-\mathrm{ATPase}$ inhibitor, suppressed water absorption in type I acini. When I. scapularis was offered a droplet of water, ticks rarely imbibed water directly $(5 \%)$, while some approached the water droplet to use the high humidity formed in the vicinity of the droplet $(23 \%)$. We conclude that during both on- and off-host stages, type I acini in salivary glands of female Ixodes scapularis absorb water and ions. 
1 Water absorption through salivary gland type I acini in the blacklegged tick, Ixodes

2 scapularis

3 Donghun Kim ${ }^{1}$, Paulina Maldonado Ruiz ${ }^{1}$, Ludek Zurek ${ }^{1}$, Yoonseong Park ${ }^{1}$

$4{ }^{1}$ Department of Entomology, Kansas State University, Manhattan, KS, USA

5

6 Corresponding Author:

7 Yoonseong Park ${ }^{1}$

8123 W. Waters Hall, Manhattan, KS, 66506-4004, USA

$9 \quad$ Email address: ypark@,ksu.edu 


\section{Abstract}

12 Tick salivary glands play critical roles in maintaining water balance for survival, as they

13 eliminate excess water and ions during blood feeding on hosts. In the long duration of fasting in

14 the off-host period, ticks secrete hygroscopic saliva into the mouth cavity to uptake atmospheric

15 water vapor. Type I acini of tick salivary glands are speculated to be involved in secretion of

16 hygroscopic saliva based on ultrastructure studies. However, we recently proposed that type I

17 acini play a role in resorption of water/ions from the primary saliva produced by other salivary

18 acini (i.e., types II and III) during the tick blood feeding phase. In this study, we tested the

19 function of type I acini in unfed female Ixodes scapularis. The route of ingested water was

20 tracked after forced feeding of water with fluorescent dye rhodamine123. We found that type-I

21 acini of the salivary glands, but not type II and III, are responsible for water uptake. In addition,

22 the ingestion of water through the midgut was also observed. Injection or feeding of ouabain, a

$23 \mathrm{Na} / \mathrm{K}$-ATPase inhibitor, suppressed water absorption in type I acini. When I. scapularis was

24 offered a droplet of water, ticks rarely imbibed water directly (5\%), while some approached the

25 water droplet to use the high humidity formed in the vicinity of the droplet $(23 \%)$. We conclude

26 that during both on- and off-host stages, type I acini in salivary glands of female Ixodes

27 scapularis absorb water and ions. 


\section{Introduction}

Maintaining water balance in terrestrial arthropods is crucial for survival. Water uptake occurs via various routes, including the anus, cuticle, air vapor, and direct drinking (Dunbar \& Winston 1975; Edney 1977; Kahl \& Knülle 1988; McMullen et al. 1976; Rudolph \& Knulle 1974). Ixodid ticks can capture atmospheric water molecules using hygroscopic saliva in the microenvironment where the relative humidity is higher than critical equilibrium activity (McMullen et al. 1976; Rudolph \& Knulle 1974). Different types of salivary gland acini have been suggested to have different roles in the water balance in Ixodid ticks (Coons et al. 1994; Kim et al. 2014; Krolak et al. 1982; Megaw \& Beadle 1979; Needham et al. 1990).

Ixodid ticks are the major vectors that transmit various pathogens causing diseases including babesiosis, anaplasmosis, and Lyme disease. In the United States, Lyme disease is the best well-known tick-borne disease transmitted by the blacklegged tick, Ixodes scapularis. In addition to their importance in human health, ticks can be an interesting model system for studying homeostasis of water. This is because of their unique ecological properties; their long blood feeding (up to two weeks) comparing to that of other blood feeding arthropods, such as mosquitoes; and their long duration of fasting (several months) when off the host. Tick salivary glands play critical roles in water balance. In female ticks, a pair of salivary glands located in the anterolateral regions of the tick body, consists of three different types of acini: types I/II/III. Type I acini are located on the anterior portion of the main salivary duct, type II acini are on the secondary branches, and type III acini are located on the most distal end tertiary branches of the salivary glands. During blood feeding, salivary glands play critical roles in eliminating excess water and ions obtained from a large amount of blood (Kaufman \& Phillips 1973; Tatchell 1967). Autocrine/paracrine dopamine signaling orchestrates an influx of solute into type-II/-III acini via 
52 dopamine receptor (D1) and efflux of solute through the salivary duct by pumping/gating the

53 acini via invertebrate D1-like dopamine receptor (InvD1L) (Kim et al. 2014; Šimo et al. 2014;

54 Šimo et al. 2011). Additionally, neuropeptides (SIFamide and MIP) innervating the basal region

55 of salivary gland acini type-II/-III are thought to be involved in controlling salivary secretion

56 (Šimo et al. 2014; Šimo et al. 2013; Šimo et al. 2009).

57 During fasting, ticks are known to secrete hygroscopic saliva into the mouth cavity to

58 uptake atmospheric water vapor and maintain the water balance (Gaede \& Knülle 1997;

59 McMullen et al. 1976; Rudolph \& Knulle 1974). In the dry environment with lower relative

60 humidity (RH) than critical equilibrium activity (CEA), secreted crystalline hygroscopic saliva is

61 then crystalized, which leads to absorption of water molecules at the RH higher than CEA, and

62 ticks then imbibe the fluid (McMullen et al. 1976; Rudolph \& Knulle 1974). Type I acini have

63 been thought to be important in this process because the size of type I acini remains constant in

64 both, off- and on-host, stages; while type-II/-III acini are enlarged in the on-host stages. In

65 addition, in an ultrastructural study, lamellate cells, cells consisting of type I acini, have

66 extensive interdigitating plasma membranes facing hemolymph and large numbers of

67 mitochondria between the infolds, which are features similar to the nasal salt glands of marine

68 birds (Doyle 1960) that produce hygroscopic solution for withdrawing atmospheric water

69 molecules. Therefore, type I acini have been generally thought to be the site of secretion for

70 hygroscopic saliva (McMullen et al. 1976; Rudolph \& Knulle 1974).

However, in this study, we found that the type I acini are indeed the site of water and ion absorption in the off-host phase, as determined by the absorption function of type I acini via

$73 \mathrm{Na} / \mathrm{K}$-ATPase in the off-host phase of female I. scapularis. In investigations of fasting ticks, we 
75 dependent on ouabain-sensitive $\mathrm{Na} / \mathrm{K}$-ATPase. 
76

77

78

79

80

81

82

83

84

85

86

87

88

89

90

91

92

93

94

95

96

97

\section{Materials \& Methods}

\section{Ticks and dehydration of ticks}

Unfed adult blacklegged ticks (I. scapularis) were obtained from the tick rearing center at Oklahoma State University (Stillwater, OK, USA). Ticks were kept in an incubator at $28^{\circ} \mathrm{C}$ and 98\% relative humidity $(\mathrm{RH})$ until the experiments. Partially dehydrated unfed female ticks were prepared by placing the ticks in the dehydration condition $\left(28^{\circ} \mathrm{C}\right.$ and $\left.\mathrm{RH} 25 \%\right)$ for 36 hours.

Feeding rhodamine123 (Rh123) and imaging fluorescence

To investigate the route of water intake in ticks, $1.28 \mu \mathrm{L}$ of $1 \mathrm{mM}$ rhodamine123 in water (Sigma-Aldrich, MO, USA) was filled in a microcapillary tube (Diameter $\sim 0.11 \mathrm{~mm}$, SigmaAldrich, MO, USA) and the tube was placed onto the mouthparts of dehydrated unfed female ticks. Ticks were allowed to ingest Rh123 under rehydration conditions (RH 98\%) at room temperature for $30 \mathrm{~min}$. After ingestion, we quantified the ingested amount and traced the locations of fluorescence signal in tick organs, specifically in the salivary glands and the gut diverticular. The volume ingested was calculated by the equation for cylinder volume $\left(\mathrm{V}=\pi \mathrm{r}^{2} \mathrm{~h}\right)$. For calculation of volume, the reduced length (h) of fluid in microcapillary tubes was measured under a grid microscope and analyzed with $\mathrm{r}^{2}=0.0127$. The locations of fluorescence in the internal organs were identified after dorsal integument of ticks was removed by a surgical scalpel. Images were captured using a camera (DFC400) attached to a stereo microscope (M205FA, Leica, Switzerland). Salivary glands were subsequently dissected out, fixed in $4 \%$ paraformaldehyde at room temperature for $30 \mathrm{~min}$, washed in PBST $(0.1 \%$ Triton X-100), and imaged on a confocal microscope (LSM700, Zeiss, Germany). 
99

100

101

102

103

104

105

106

107

108

109

110

111

112

113

114

115

116

117

118

119

Injection/pre-ingestion of ouabain or Hank's saline buffer

To investigate the physiological function of $\mathrm{Na} / \mathrm{K}$-ATPase, we injected or orally introduced ouabain as a Na/K-ATPase blocker and Hank's saline buffer as control. We injected $50 \mathrm{~nL}$ of $100 \mu \mathrm{M}$ ouabain (Sigma Aldrich, MO, USA) or Hank's saline into dehydrated (36 hr) unfed female ticks with a Nanoliter 2010 injector controlled by Micro4 (WPI, FL, USA). Ticks injected by either ouabain or Hank's saline were placed in dehydration conditions for an additional $30 \mathrm{~min}$; then, these ticks were subsequently fed fluid containing $1 \mathrm{mM} \mathrm{Rh} 123$ in a microcapillary tube under the rehydration conditions in a water saturated glass jar (RH 98\%) for $30 \mathrm{~min}$.

For pre-ingestion experiments, dehydrated unfed female ticks were offered ouabain (100 $\mu \mathrm{M})$ or Hank's saline in a microcapillary tube for $30 \mathrm{~min}$. After the 30 -min pre-ingestion of ouabain (varied between 0.1 and $0.15 \mu \mathrm{L}$ ), the micropipettes were replaced by micropipettes filled with Rh123 under the rehydration condition for an additional $30 \mathrm{~min}$. After injection and pre-ingestion experiments, the ingested volume and fluorescence signal were quantified and imaged as described in a previous section.

\section{Natural water drinking of dehydrated unfed female ticks}

To examine whether questing I. scapularis drink water, dehydrated and unfed female ticks were exposed to $5 \mu \mathrm{L}$ of water in the center of a $50 \mathrm{~mm} \times 9 \mathrm{~mm}$ airtight petri dish (Falcon, NY, USA) at room temperature for 1 hour. We counted the number of ticks that approached and ingested the natural water through their mouthparts. 
121 Statistical analyses

122 The significant difference of each experiment was found with either a Student's t-test

$123(\mathrm{p}=0.05)$ or chi-square test. The t-test was used to analyze statistical differences between control

124 and experimental groups of Rh123 ingestion assays ( $\mathrm{n}=25$ total), pre-ingestion assays ( $\mathrm{n}=11$ in

125 each treatment), and injection assays ( $\mathrm{n}=5$ in each treatment). The Chi-square test was used to

126 analyze the frequency of Rh123 fluorescence of type I acini from pre-ingestion assays with

127 Hank's saline buffer and ouabain. 


\section{Results and Discussion}

130

131

132

133

134

135

136

137

138

139

140

141

142

143

144

145

146

147

148

149

150

Water absorption through type I acini of salivary glands

Studies of the ultrastructure of type I acini have suggested that type I acini play a critical role in the secretion of hygroscopic saliva to capture atmospheric water vapor (Binnington 1978;

Kahl \& Alidousti 1997; McMullen et al. 1976; Megaw \& Beadle 1979; Needham et al. 1990). In this process, the type I acini are generally thought to be the direct site for producing hygroscopic saliva (Krolak et al. 1982; Megaw \& Beadle 1979; Needham et al. 1990), while the function of the salivary glands and midgut for direct water absorption is speculated as the site to uptake water (McMullen et al. 1976). However, a study focused on ultrastructural changes of type I acini in different humidities contradicts the function of type I acini in direct production of hygroscopic saliva. Dehydration of ticks results in an orthodox configuration (inactive form) of mitochondria, while rehydration changed the mitochondria to a condensed configuration (active form in rehydration) in the type I lamellate cells (Needham et al. 1990; Needham \& Coons 1984). This is in contradiction to the prediction that type I acini participate in active production of hygroscopic saliva. In this study, we provide clear evidence that type I acini function in direct water absorption.

To visualize the routes of water absorption in fasting ticks, we performed forced feeding of fluorescent tracer Rh123 for 30 min using a microcapillary tube on dehydrated unfed female ticks. There were large individual variations in the ingested amount of solution in the range of $0.052-0.448 \mu \mathrm{L}$. The majority of the tested individuals $(64 \%)$ were positive for fluorescence in both cell bodies of type I acini (but not in type II/III acini) and the gut diverticular (Fig. 1A and Fig. 2), while other individuals (36\%) with low amount of ingestion $(<0.15 \mu \mathrm{L})$ lacked 
151 fluorescence in type I acini (Fig. 1B), but presented only in the gut diverticula (Fig. 1B and Fig. 152 2).

This result was similar to our previous observation (Kim et al. 2016) with some important

154 differences. The previous study showed all tested ticks were positive for fluorescence in the type I acini, while some lacked fluorescence in the midgut, which is in contrast to the results in this study with partially positive type I acini. We speculated that the difference was caused by two reasons, the degree of dehydration of the ticks ( $12 \mathrm{~h} \mathrm{vs.} 36 \mathrm{~h}$ in this study) and the longer feeding duration (immediately after the drinking water ( $<15$ min vs. 30 min drinking in this study). Long dehydration times likely resulted in inactivation of the type I acini in some individual ticks, considering it was demonstrated with the inactive form of mitochondria in type I acini found in $24 \mathrm{~h}$ dehydrated ticks (Needham et al. 1990). Longer pre-conditioning of dehydration and a longer duration of forced-drinking in this study compared to previous studies likely allowed for drinking through the gut, while some individuals lacked water uptake through the salivary glands.

In individuals that ingested high levels of rhodamine123, fluorescence was not only found in the type I acini and gut but it also permeated to the hemolymph. This observation suggests that the ticks actively uptake water through type I acini. In addition, the presence of tracer in the cytoplasm of type I acini cells and the hemolymph implies that the absorption mechanism likely includes active transport for the tracer dye Rh123, which is often used for experiments testing the roles of the membrane transporter (Forster et al. 2012; Jancis et al. 1993).

\section{$\mathrm{Na} / \mathrm{K}$-ATPase in water absorption}

We tested the role of $\mathrm{Na} / \mathrm{K}$-ATPase in water absorption using ouabain, which is a wellknown Na/K-ATPase inhibitor. Previous studies described abundant Na/K-ATPase 
174 immunoreactivity in the type I acini (Kim et al. 2016; Needham et al. 1990). Initially, we

175 injected ouabain in the hemocoel where it likely inhibits $\mathrm{Na} / \mathrm{K}$-ATPases in the whole body. The

176 injection was followed by forced feeding of Rh123 through a microcapillary tube to quantify the

177 ingested volume and observe the fluorescence of salivary glands.

Ouabain injection significantly reduced the volume of water ingestion (Fig. 3). The

179 ingested volume of ouabain-injected ticks was $28 \%$ of Hank's saline-injected ticks ( $\mathrm{n}=5$ for each). Most of the type I acini lacked tracer, which was only observed from the main duct in ouabain injections (Fig. 4B), while Hank's saline injections showed Rh123 fluorescence in the type I acini (Fig. 4A).

To achieve more specific inhibition of $\mathrm{Na} / \mathrm{K}$-ATPase in the type I acini of salivary glands, we treated ticks with ouabain by pre-ingestion. Therefore, pre-ingestion of ouabain for $30 \mathrm{~min}$ (uptaken volume varied between 0.1 and $0.15 \mu \mathrm{L}$ ) affected $\mathrm{Na} / \mathrm{K}$-ATPase in water uptake by type I acini and the midgut specifically. Ticks with ouabain pre-ingestion consumed $67 \%$ of the than those with pre-ingestion of Hank's saline (Fig. 5B; $p=0.004$ ). Although the effect of preingestion of ouabain on type I-mediated water uptake was less pronounced than the effect shown in the injection experiment, this was presumably due to the low dose reaching the target tissue. The broad pharmacological effects of ouabain may also include consequences of mitochondrial calcium deficiency or impairment of mitochondrial energy metabolism, which are also known activities of ouabain (Liu et al. ; Roevens \& de Chaffoy de Courcelles 1990), in addition to the inhibitory effects on $\mathrm{Na} / \mathrm{K}-\mathrm{ATPase}$ in type I acini by the ouabain pre-ingestion. This result supports the significant roles of $\mathrm{Na} / \mathrm{K}$-ATPase in the type I acini-mediated water uptake. 

both, the gut diverticular space and salivary glands. Dehydration levels of ticks influenced the route of water absorption via gut diverticular spaces and salivary glands or gut diverticular spaces only. Under severe dehydration, $\mathrm{Na} / \mathrm{K}$-ATPase mediated water uptake in type I acini might not be functional due to the inactive form of mitochondria (Needham et al. 1990). water uptake in type I acini.

Natural behaviors in water uptake

Finally, we investigated whether I. scapularis voluntarily drink water. We observed several different patterns of behavior when a water droplet was offered. The majority of dehydrated ticks were not attracted to water and randomly moved or stayed away from the water drop (72\%, 31/43, Fig. 6A). However, some dehydrated ticks actively approached and stayed close to the water drop without directly contacting the water with their mouthparts $(23 \%, 10 / 43$, Fig. $6 \mathrm{C}$ and D). These ticks displayed two patterns: i) they spread the front pair of legs toward the water drop and the extended legs and often directly touched the water drop (Fig. 6C); and ii) they folded their first two pairs of legs and stayed close to the water drop (Fig. 6D). A small group of dehydrated ticks actively approached the water drop, placed the chelicera on top of the water surface, and drank the water (5\%, 2/43, Fig. 6B and Video S1). In the third case, pulsatile water flow was observed between hypostome and chelicerates (Video S1). 
220 the earlier study of I. ricinus and I. scapularis (Kahl \& Knülle 1988; Lees 1946; Yoder \&

221 Spielman 1992), under our experimental conditions, I. scapularis directly drank water in rare 222 occasions $(5 \%)$.

223 A model for the roles of type I acini in water balance

A previous study indicated that on-host ticks actively excreted an excess of salt and water through salivary glands by producing iso/hypo osmotic saliva (Kim et al. 2016). Sodium-rich primary saliva was produced by a dopamine-mediated electrochemical gradient in type II and III acini. In that study and the present study, immunohistochemistry revealed $\mathrm{Na} / \mathrm{K}$-ATPase in type II/III in addition to type I acini, indicating $\mathrm{Na} / \mathrm{K}$-ATPase was the major source for the electrochemical gradient in the formation of primary saliva-(Fig. 7A). Based on the results of this study in the fasting tick, we propose that hygroscopic hyperosmolar saliva is formed as a result of shutting down the absorptive function of type I acini under severely dry conditions (Fig. 7B). Therefore, the hyperosmolar primary saliva formed from type II/III acini in the previous study was directly secreted without the $\mathrm{Na} / \mathrm{K}$-ATPase-mediated resorptive function of type I acini (Kim et al. 2016). Once water molecules are captured in the hygroscopic saliva, type I acini are the site for absorbing water from the diluted saliva (Fig. 7C). The ions (mainly $\mathrm{Na}^{+}$) used for generation of electrochemical gradient for water uptake in type I acini are likely recycled for secretory activity in the type II/III acini. insects (i.e., the American cockroach) (Hille \& Walz 2008). Na/K-ATPase in the apical surface of acinar peripheral cells in the insect is similar to that in the apical surface of types II and III acini in ticks (Kim et al. 2016). The resorptive function of type I acini with $\mathrm{Na} / \mathrm{K}$-ATPase in the

242 basolateral infolding is the same as the duct cells in insect salivary glands with a similar 
243 subcellular location of $\mathrm{Na} / \mathrm{K}$-ATPase. This configuration facilitates the production of primary

244 saliva in the distal part of salivary glands (types II and III in tick and peripheral cells in the

245 American cockroach), which is followed by resorption of ion/water in the proximal part of the

246 salivary glands (type I and duct).

A previous study successfully showed the phenotype for RNAi of $\mathrm{Na} / \mathrm{K}-\mathrm{ATP}$ ase that

248 resulted in failure in the full engorgement and reduced egg numbers in oviposition (Karim et al.

249 2008). Based on the RNAi of $\mathrm{Na} / \mathrm{K}$-ATPase in our laboratory followed by accessing the degree

250 of suppression using real time PCR and immunohistochemical staining of $\mathrm{Na} / \mathrm{K}$-ATPase, partial

251 suppression of $\mathrm{Na} / \mathrm{K}$-ATPase from synganglion and salivary glands was associated with

252 phenotype failure in full engorgement. However, the immunohistochemistry of $\mathrm{Na} / \mathrm{K}$-ATPase

253 suggested that knock down of $\mathrm{Na} / \mathrm{K}$-ATPase immunoreactivities in type I acini did not occur,

254 while partial knock down of the immunoreactivities in types II/III acini were observed (Fig. S1-

255 2). Our RNAi results implied that knocking down a gene product could be also tissue/target

256 specific, and suggested that type I acini Na/K-ATPase was difficult to knock down by RNAi due

257 to the long half-life of the protein.

259 previous studies. Our study is important in: a) identifying a route for drug delivery that may be useful for physiological studies, b) uncovering a novel mechanism of water absorption in ticks and that may be also common in other arachnid species, and c) application of this new

262 knowledge for tick management.

\section{Acknowledgements}


266

267

268

269

270

271

272

273

274

275

276

277

278

279

280

281

282

283

284

285

286

287

288

289

290

291

292

293

294

295

296

297

298

299

300

301

302

303

304

305

306

307

308

\section{Figure 1.}

Fluorescence in gut diverticular and salivary glands after feeding water with the tracer dye rhodamine 123 (Rh123). (A) Example of fluorescence positive in both salivary glands and gut diverticular. (B) Example of fluorescence positive only in gut diverticular. Empty arrow heads indicate gut diverticular. Solid arrow head indicates salivary glands. Asterisks indicate autofluorescence of hindgut and rectal sac, which were confirmed in previous observation (Kim et al. 2016). Scale bar equals $0.5 \mathrm{~mm}$.

\section{Figure 2.}

Consumed volume of water containing rhodamine 123 (Rh123) after a forced feeding for 30 min. The consumed volume is compared for the ticks with the fluorescence and without fluorescence in the type I acini. Each symbol indicates ingested amount of Rh123 of individual dehydrated female I. scapularis tick after a forced feeding for $30 \mathrm{~min}(\mathrm{n}=25)$. Boxes indicate range data from $25 \%$ to $75 \%$. The horizontal line in the box is for median and the line crossing the box is mean. Whiskers with lines indicate $99 \%$ and $1 \%$ of data. The significant difference $(\mathrm{p}=0.05$, asterisk) was found in Student T-test.

\section{Figure 3}

Reduction in the volume of consumed water after ouabain injection. Total ingested amount of water containing Rh123 for 30 min were compared between ticks injected either by Hank's saline $(n=5)$ or ouabain $(n=5)$. Each symbol indicates ingested volume of individual dehydrated female $I$. scapularis tick. Boxes indicate data range from $25 \%$ to $75 \%$. The horizontal line in the box is for median and the line crossing the box is mean. Whiskers with lines indicate $99 \%$ and $1 \%$ of data. The significant difference $(\mathrm{p}=0.05$, asterisk) was found in Student T-test.

\section{Figure 4}

Tick salivary glands showing fluorescence in the type I acini after a forced feeding of water containing rhodamine 123 (Rh123). Green and blue colors indicate rhodamine 123 and nuclei, respectively. (A) Salivary gland from Hank's saline-injected ticks displayed green fluorescence in the type-I acini. (B) Salivary gland from ouabain-injected ticks lacked green fluorescence in the type-I acini. Scale bars indicate $100 \mu \mathrm{m}$.

\section{Figure 5}

Effects of ouabain pre-ingestion on the absorption function of type I acini. (A) Amount ingested by the ticks that pre-ingested Hank's saline $(n=11)$ and ouabain $(n=11)$. Each symbol indicates ingested volume of rhodamine 123 of individual dehydrated female $I$. scapularis. Boxes indicate data range from $25 \%$ to $75 \%$. The horizontal line in the box is for median and the line crossing the box is mean. Whiskers with lines indicate $99 \%$ and $1 \%$ of data. The significant difference ( $\mathrm{p}=0.05$, asterisk) was found in Student T-test. (B) Comparison fluorescence observed from type-I acini between pre-ingestion with Hank's saline and ouabain. The data were analyzed by Chi-Squared test $(p=0.004)$.

\section{Figure 6}


Three different patterns of behavior observed from the dehydrated unfed female $I$. scapularis when a water drop is offered. (A) Percent of different tick behavioral patterns; No attraction, Attracted \& Stayed, and Drink water in pie chart. (B) Example of ticks drinking water via mouthpart. (C and D) Two sub-patterns of attracted \& stayed, respectively. (C) Spreading the front pair of legs toward water drop and directly touching water drop. (D) Folding first two pairs of legs and stayed close to water drop.

\section{Figure 7}

A model proposed for the function of type I acini in water absorption. (A) During blood feeding, ticks secrete iso/hyposmotic saliva. Type II and III acini produce hyperosmotic primary saliva, and type-I acini subsequently reabsorb ions immediately before secretion. (B) During fasting in vegetation, ticks secrete hyperosmotic saliva to uptake water vapor from subsaturated air. Hyperosmotic saliva is mainly produced by type II and III acini. Nonfunctional type I acini is shown by gray X mark. (C) During fasting in dehydrated condition, captured water from air vapor is absorbed via type I acini, while type II and III acini have no function in water absorption. The acini figure is modified from Binnington (1978).

\section{Supplementary data legend}

\section{Video S1.}

Direct water drinking observed in a dehydrated unfed female $I$. scapularis tick. Video record showing a tick drinking water through the mouth cavity between hypostome and chelicerates. Chelicerates cover half of hypostome but not all. Pulsatile movement of water flow is shown in the cavity filled with water by capillary force.

Figure S1. Na/K-ATPase immunoreactivities were observed in type I/II/III of Hank's saline buffer injected unfed female salivary glands. Arrow heads indicated immunoreactivities of Na/K-ATPase. Red indicated positive immunoreactivities of $\mathrm{Na} / \mathrm{K}$-ATPase. Overview image of salivary glands (A). Close image focusing on type II and III acini (B \& C). Scale bar indicated $50 \mathrm{um}$.

Figure S2. Na/K-ATPase immunoreactivities were observed in only type I of dsRNA-Na/K-ATPase injected unfed female salivary glands. Arrow heads indicated immunoreactivities of $\mathrm{Na} / \mathrm{K}-\mathrm{ATPase}$. Red indicated positive immunoreactivities of $\mathrm{Na} / \mathrm{K}$-ATPase. Overview image of salivary glands (A). Close image focusing on type II and III acini (B \& C). Scale bar indicated $50 \mathrm{um}$. 
342

343

344

345

346

347

348

349

350

351

352

353

354

355

356

357

358

359

360

361

362

363

364

365

366

367

368

369

370

371

372

373

374

375

376

377

378

379

380

381

382

383

384

385

\section{References}

Binnington KC. 1978. Sequential changes in salivary gland structure during attachment and feeding of the cattle tick, Boophilus microplus. International Journal for Parasitology 8:97-115. http://dx.doi.org/10.1016/0020-7519(78)90004-8

Coons LB, Lessman CA, Ward MW, Berg RH, and Lamoreaux WJ. 1994. Evidence of a myoepithelial cell in tick salivary glands. International Journal for Parasitology 24:551-562.

Doyle WL. 1960. The principal cells of the salt-gland of marine birds. Experimental Cell Research 21:386393. http://dx.doi.org/10.1016/0014-4827(60)90270-6

Dunbar BS, and Winston PW. 1975. The site of active uptake of atmospheric water in larvae of Tenebrio molitor. Journal of Insect Physiology 21:495-500. http://dx.doi.org/10.1016/00221910(75)90154-7

Edney EB. 1977. Uptake of Liquid Water. In: Edney EB, ed. Water Balance in Land Arthropods. Berlin, Heidelberg: Springer Berlin Heidelberg, 172-188.

Forster S, Thumser AE, Hood SR, and Plant N. 2012. Characterization of Rhodamine-123 as a Tracer Dye for Use In In vitro Drug Transport Assays. PloS One 7:e33253. 10.1371/journal.pone.0033253

Gaede K, and Knülle W. 1997. On the mechanism of water vapour sorption from unsaturated atmospheres by ticks. Journal of Experimental Biology 200:1491-1498.

Hille C, and Walz B. 2008. Characterisation of neurotransmitter-induced electrolyte transport in cockroach salivary glands by intracellular $\mathrm{Ca} 2+, \mathrm{Na}+$ and $\mathrm{pH}$ measurements in duct cells. Journal of Experimental Biology 211:568-576. 10.1242/jeb.010207

Jancis EM, Hong-xing C, Carbone R, Hochberg RB, and Dannies PS. 1993. Rapid stimulation of rhodamine 123 efflux from multidrug-resistant KB cells by progesterone. Biochemical Pharmacology 46:1613-1619. http://dx.doi.org/10.1016/0006-2952(93)90331-P

Kahl O, and Alidousti I. 1997. Bodies of liquid water as a source of water gain for Ixodes ricinus ticks (Acari: Ixodidae). Experimental and Applied Acarology 21:731-746. 10.1023/A:1018469021161

Kahl O, and Knülle W. 1988. Water vapour uptake from subsaturated atmospheres by engorged immature ixodid ticks. Experimental and Applied Acarology 4:73-83. 10.1007/bf01213843

Karim S, Kenny B, Troiano E, and Mather T. 2008. RNAi-mediated gene silencing in tick synganglia: A proof of concept study. BMC Biotechnology 8:30.

Kaufman WR, and Phillips JE. 1973. Ion and Water-Balance in Ixodid Tick Dermacentor-Andersoni .1. Routes of Ion and Water Excretion. Journal of Experimental Biology 58:523-536.

Kim D, Šimo L, and Park Y. 2014. Orchestration of salivary secretion mediated by two different dopamine receptors in the blacklegged tick Ixodes scapularis. Journal of Experimental Biology 217:36563663.

Kim D, Urban J, Boyle DL, and Park Y. 2016. Multiple functions of Na/K-ATPase in dopamine-induced salivation of the Blacklegged tick, Ixodes scapularis. Scientific Reports 6:21047. 10.1038/srep21047. http://www.nature.com/articles/srep21047\#supplementary-information

Krolak JM, Ownby CL, and Sauer JR. 1982. Alveolar structure of salivary glands of the lone star tick, Amblyomma americanum (L.): unfed females. Journal of Parasitology 68:61-82.

Lees AD. 1946. The water balance in Ixodes ricinus L. and certain other species of ticks. Parasitology 37:1-20. 10.1017/S0031182000013093

Liu T, Brown DA, and O'Rourke B. Role of mitochondrial dysfunction in cardiac glycoside toxicity. Journal of Molecular and Cellular Cardiology 49:728-736. 10.1016/j.yjmcc.2010.06.012 
403

404

405

406

407

408

409

410

411

412

413

414

415

416

417

418
McMullen HL, Sauer JR, and Burton RL. 1976. Possible role in uptake of water vapour by ixodid tick salivary glands. Journal of Insect Physiology 22:1281-1285. http://dx.doi.org/10.1016/00221910(76)90107-4

Megaw MJW, and Beadle DJ. 1979. Structure and function of the salivary glands of the tick, Boophilus microplus canestrini (Acarina : Ixodidae). International Journal of Insect Morphology and Embryology 8:67-83. http://dx.doi.org/10.1016/0020-7322(79)90007-2

Needham G, Rosell R, Greenwald L, and Coons LB. 1990. Ultrastructure of type-I salivary-gland acini in four species of ticks and the influence of hydration states on the type-I acini ofAmblyomma americanum. Experimental and Applied Acarology 10:83-104. 10.1007/BF01194085

Needham GR, and Coons LB. 1984. Ultrastructural changes in type I alveoli of the salivary glands from hydrating and desiccating lone star ticks.

Roevens P, and de Chaffoy de Courcelles D. 1990. Ouabain increases the calcium concentration in intracellular stores involved in stimulus-response coupling in human platelets. Circulation Research 67:1494.

Rudolph D, and Knulle W. 1974. Site and mechanism of water vapour uptake from the atmosphere in ixodid ticks. Nature 249:84-85.

Šimo L, Koči J, Kim D, and Park Y. 2014. Invertebrate specific D1-like dopamine receptor in control of salivary glands in the black-legged tick Ixodes scapularis. Journal of Comparative Neurology 522:2038-2052. 10.1002/cne.23515

Šimo L, Koči J, and Park Y. 2013. Receptors for the neuropeptides, myoinhibitory peptide and SIFamide, in control of the salivary glands of the blacklegged tick Ixodes scapularis. Insect Biochemistry and Molecular Biology 43:376-387. http://dx.doi.org/10.1016/j.ibmb.2013.01.002

Šimo L, Koci J, Zitnan D, and Park Y. 2011. Evidence for D1 dopamine receptor activation by a paracrine signal of dopamine in tick salivary glands. PloS One 6:e16158. 10.1371/journal.pone.0016158

Šimo L, Žitňan D, and Park Y. 2009. Two novel neuropeptides in innervation of the salivary glands of the black-legged tick, Ixodes scapularis: Myoinhibitory peptide and SIFamide. Journal of Comparative Neurology 517:551-563. 10.1002/cne.22182

Tatchell RJ. 1967. Salivary Secretion in the Cattle Tick as a Means of Water Elimination. Nature 213:940941.

Yoder JA, and Spielman A. 1992. Differential capacity of larval deer ticks (Ixodes dammini) to imbibe water from subsaturated air. Journal of Insect Physiology 38:863-869. http://dx.doi.org/10.1016/0022-1910(92)90097-W 


\section{Figure 1}

Fluorescence in gut diverticular and salivary glands after feeding water with the tracer dye rhodamine 123 (Rh123).

(A) Example of fluorescence positive in both salivary glands and gut diverticular. (B) Example of fluorescence positive only in gut diverticular. Empty arrow heads indicate gut diverticular. Solid arrow head indicates salivary glands. Asterisks indicate auto-fluorescence of hindgut and rectal sac, which were confirmed in previous observation (Kim et al. 2016). Scale bar equals $0.5 \mathrm{~mm}$.

*Note: Auto Gamma Correction was used for the image. This only affects the reviewing manuscript. See original source image if needed for review.

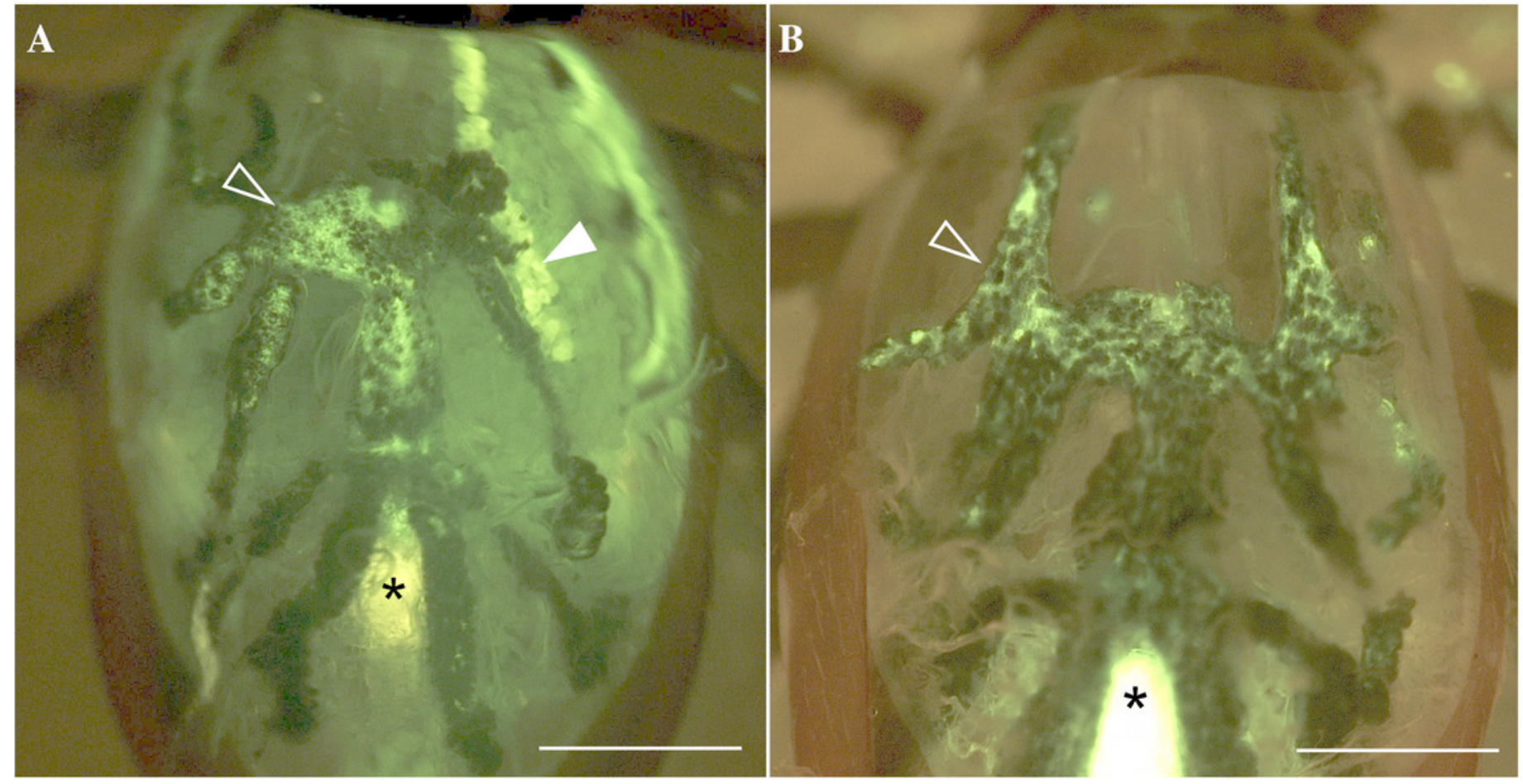


Figure 2

Consumed volume of water containing rhodamine 123 (Rh123) after a forced feeding for $30 \mathrm{~min}$.

The consumed volume is compared for the ticks with the fluorescence and without fluorescence in the type I acini. Each symbol indicates ingested amount of Rh123 of individual dehydrated female I. scapularis tick after a forced feeding for $30 \mathrm{~min}(\mathrm{n}=25)$. Boxes indicate range data from $25 \%$ to $75 \%$. The horizontal line in the box is for median and the line crossing the box is mean. Whiskers with lines indicate $99 \%$ and $1 \%$ of data. The significant difference ( $p=0.05$, asterisk) was found in Student T-test.

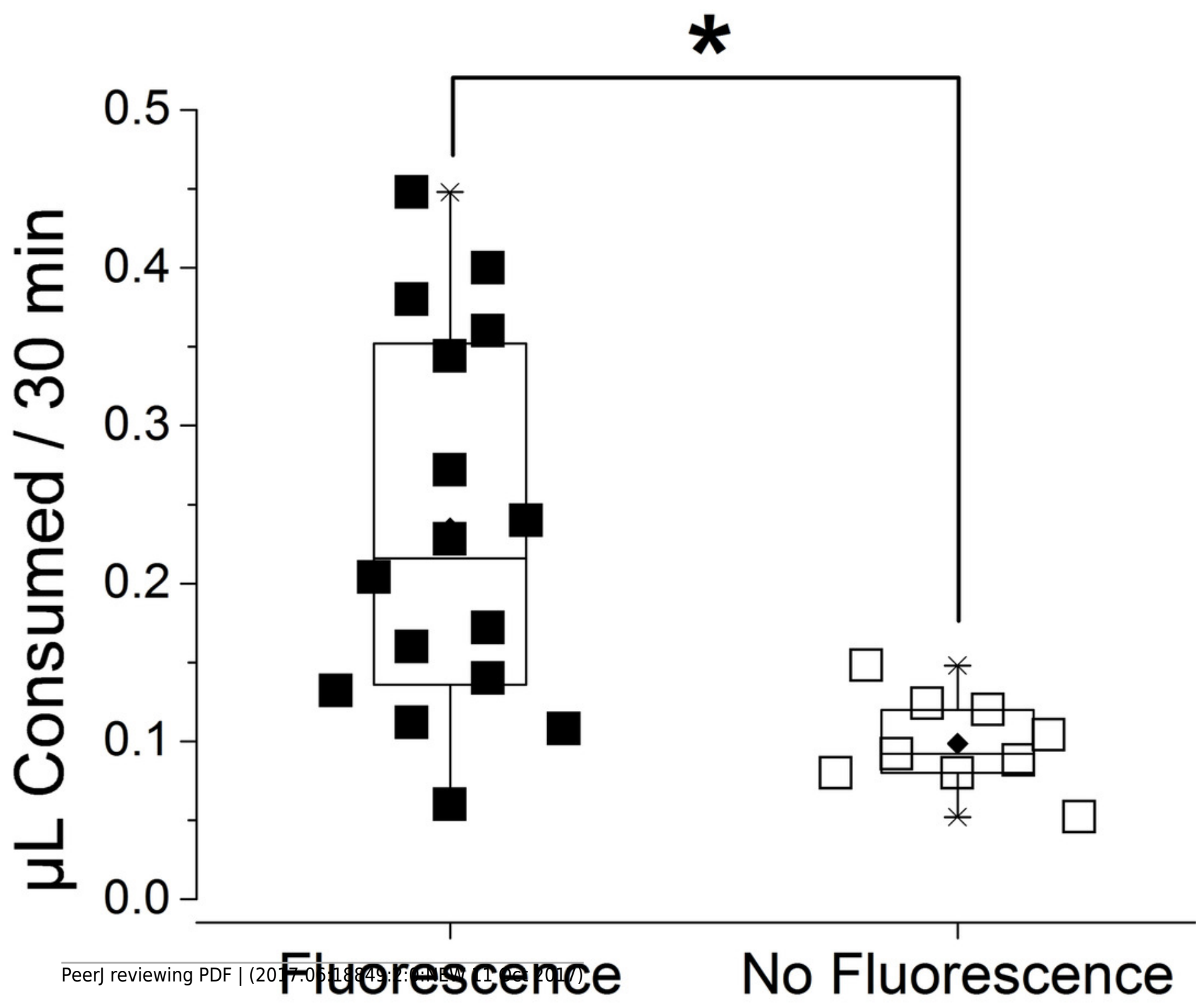




\section{Figure 3}

Reduction in the volume of consumed water after ouabain injection.

Total ingested amount of water containing Rh123 for 30 min were compared between ticks injected either by Hank's saline $(n=5)$ or ouabain $(n=5)$. Each symbol indicates ingested volume of individual dehydrated female I. scapularis tick. Boxes indicate data range from $25 \%$ to $75 \%$. The horizontal line in the box is for median and the line crossing the box is mean. Whiskers with lines indicate $99 \%$ and $1 \%$ of data. The significant difference $(p=0.05$, asterisk) was found in Student T-test. 


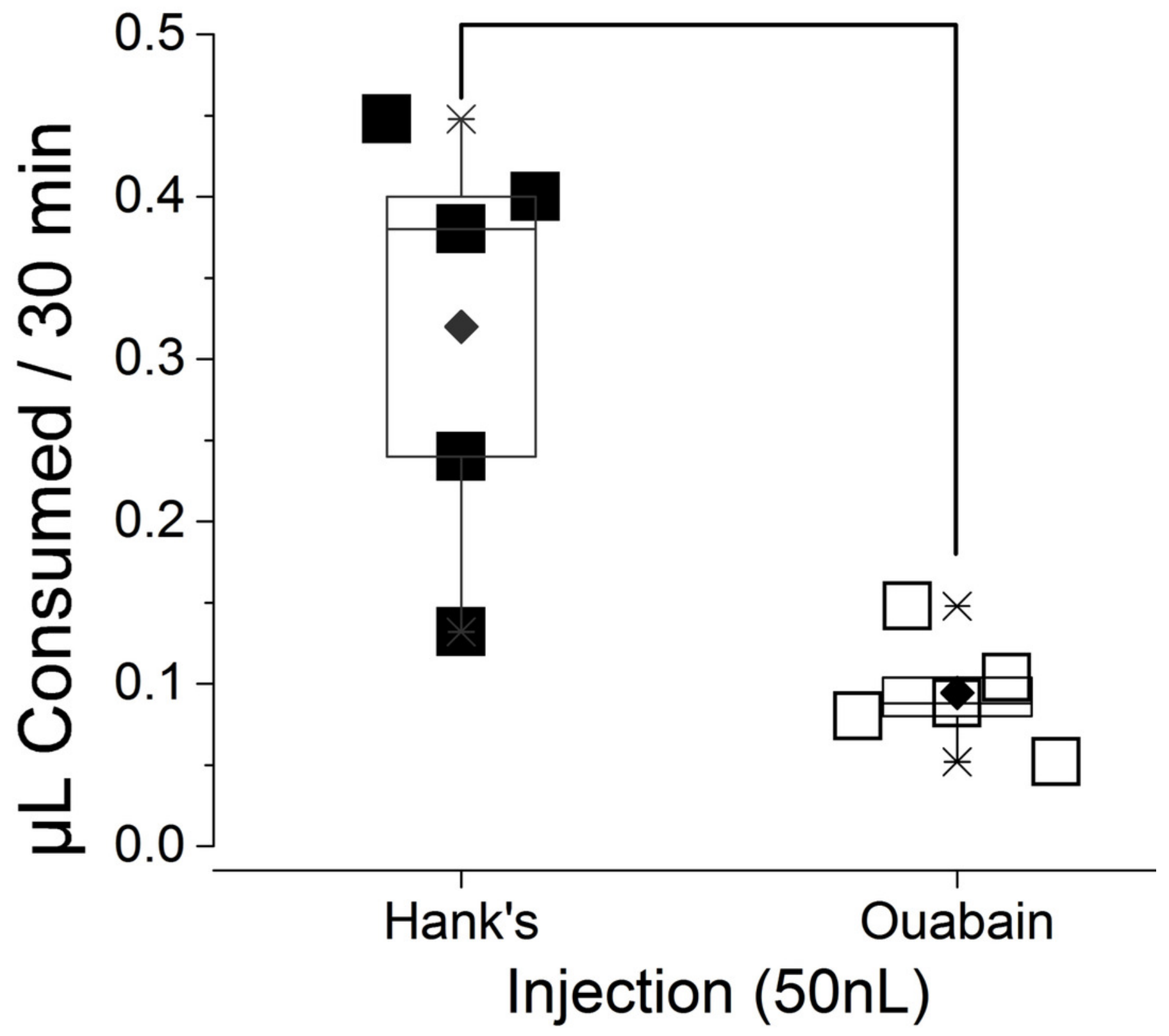




\section{Figure 4}

Tick salivary glands showing fluorescence in the type I acini after a forced feeding of water containing rhodamine 123 (Rh123).

Green and blue colors indicate rhodamine 123 and nuclei, respectively. (A) Salivary gland from Hank's saline-injected ticks displayed green fluorescence in the type-I acini. (B) Salivary gland from Ouabain-injected ticks lacked green fluorescence in the type-I acini. Scale bars indicate $100 \mu \mathrm{m}$.

*Note: Auto Gamma Correction was used for the image. This only affects the reviewing manuscript. See original source image if needed for review.

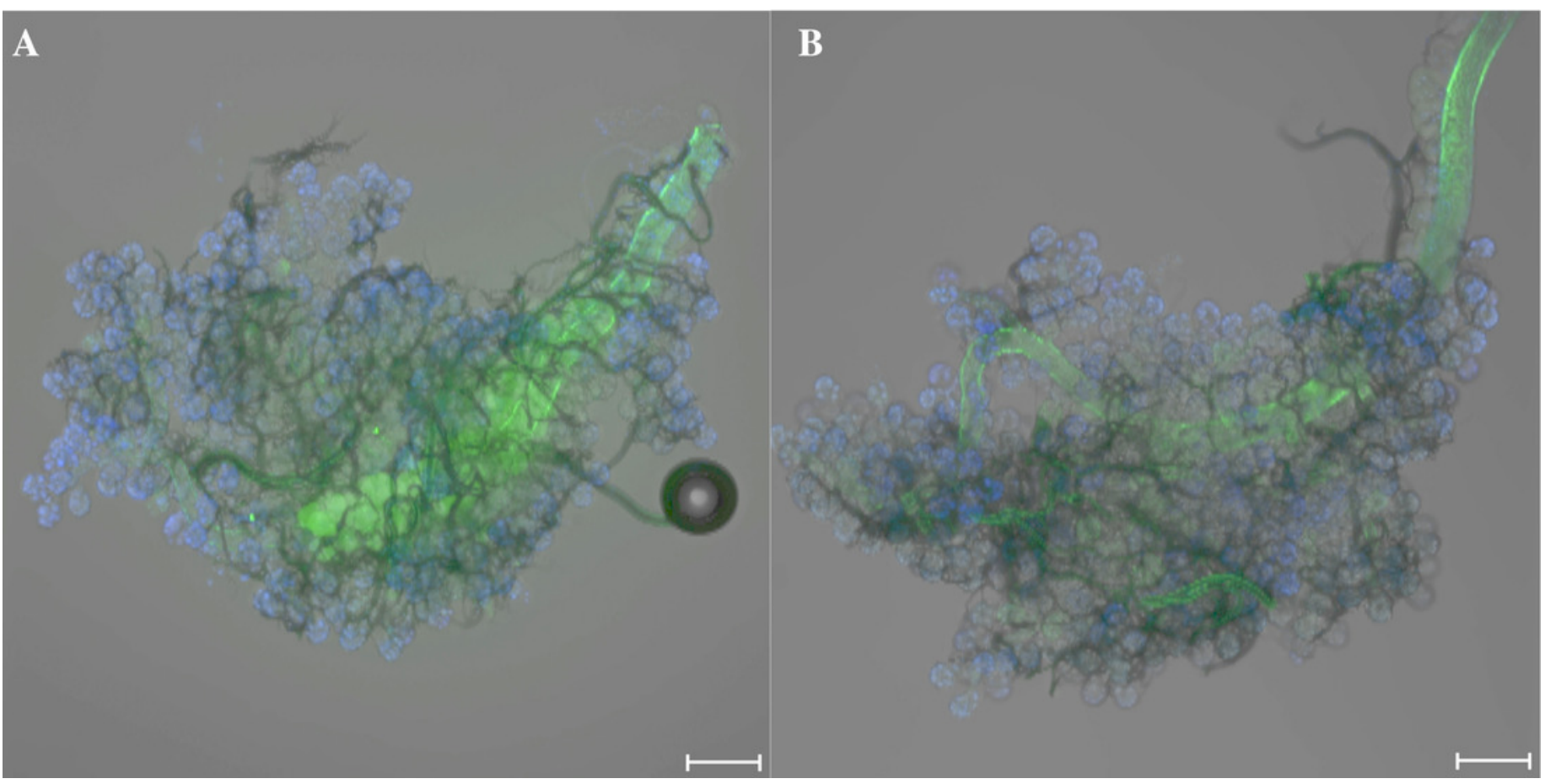




\section{Figure 5}

Effects of ouabain pre-ingestion on the absorption function of type I acini.

(A) Amount ingested by the ticks that pre-ingested Hank's saline $(n=11)$ and ouabain $(n=11)$.

Each symbol indicates ingested volume of rhodamine 123 of individual dehydrated female $I$. scapularis. Boxes indicate data range from $25 \%$ to $75 \%$. The horizontal line in the box is for median and the line crossing the box is mean. Whiskers with lines indicate $99 \%$ and $1 \%$ of data. The significant difference ( $p=0.05$, asterisk) was found in Student T-test. (B) Comparison fluorescence observed from type-I acini between pre-ingestion with Hank's saline and ouabain. The data were analyzed by Chi-Squared test $(p=0.004)$.
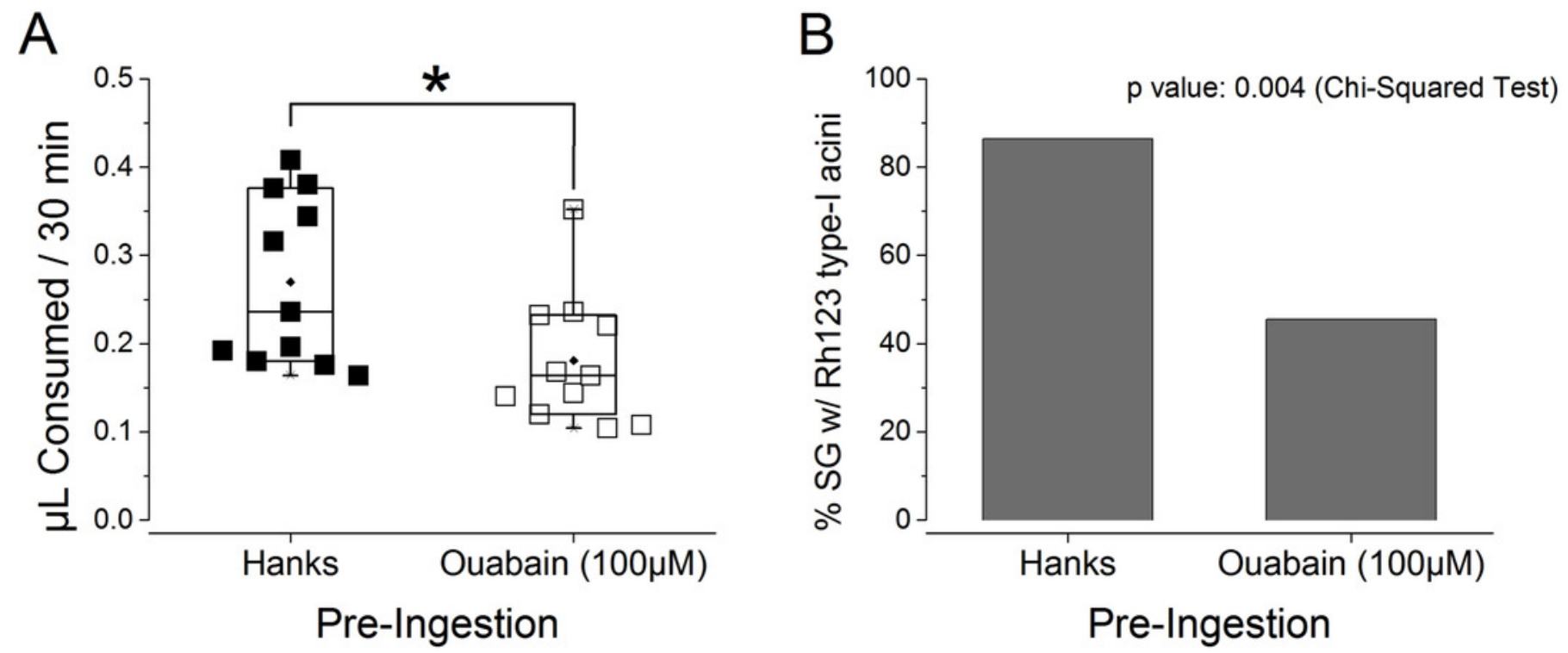


\section{Figure 6}

Three different patterns of behavior observed from the dehydrated unfed female $I$. scapularis when a water drop is offered.

(A) Percent of different tick behavioral patterns; No attraction, Attracted \& Stayed, and Drink water in pie chart. (B) Example of ticks drinking water via mouthpart. (C and D) Two subpatterns of attracted \& stayed, respectively. (C) Spreading the front pair of legs toward water drop and directly touching water drop. (D) Folding first two pairs of legs and stayed close to water drop.

A
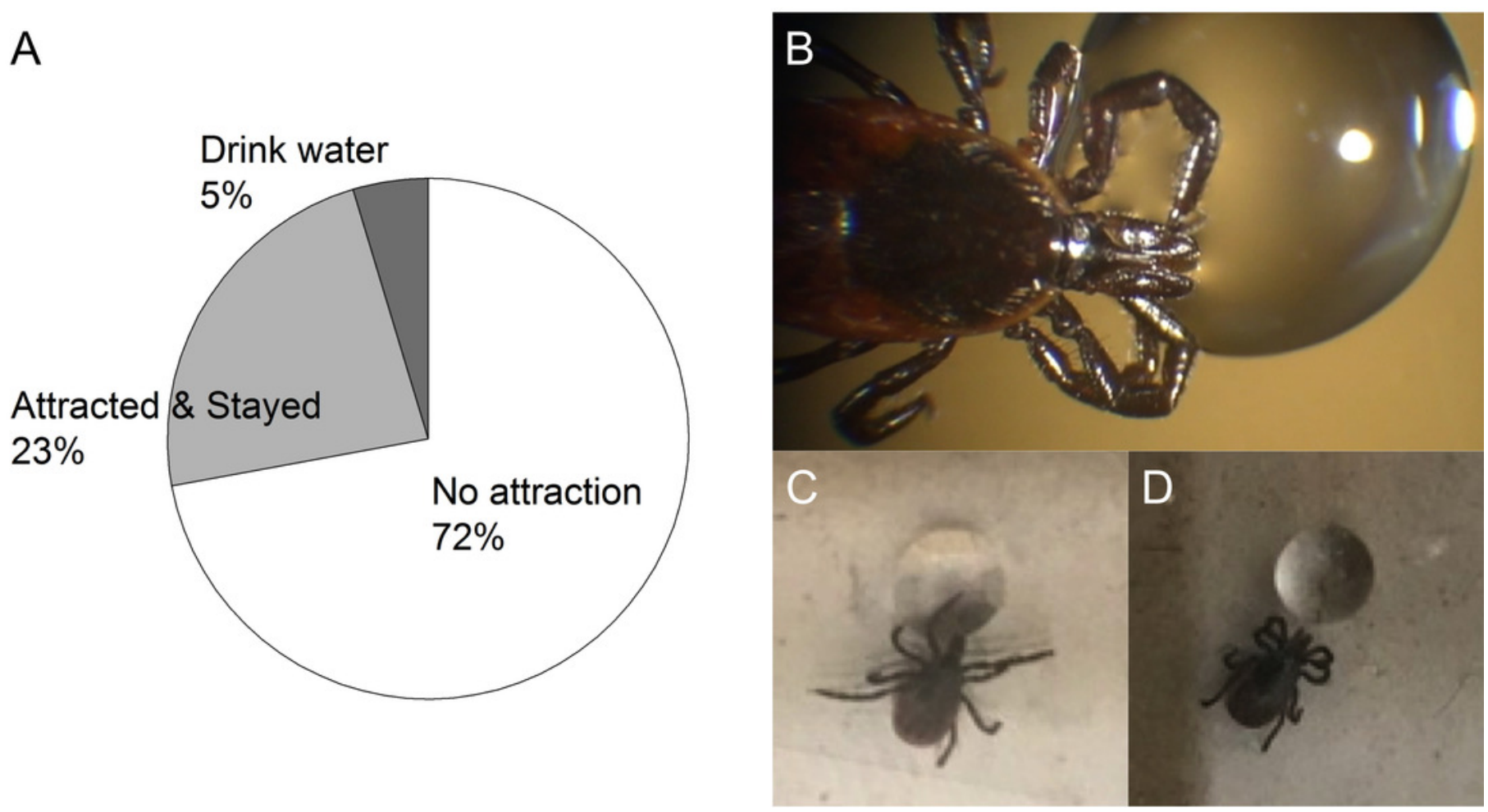


\section{Figure 7}

A model proposed for the function of type I acini in water absorption.

(A) During blood feeding, ticks secrete iso/hyposmotic saliva. Type II and III acini produce hyperosmotic primary saliva, and type-I acini subsequently reabsorb ions immediately before secretion. (B) During fasting in vegetation, ticks secrete hyperosmotic saliva to uptake water vapor from subsaturated air. Hyperosmotic saliva is mainly produced by type II and III acini. Nonfunctional type I acini is shown by gray $\mathrm{X}$ mark. (C) During fasting in dehydrated condition, captured water from air vapor is absorbed via type I acini, while type II and III acini have no function in water absorption. The acini figure is modified from Binnington (1978).

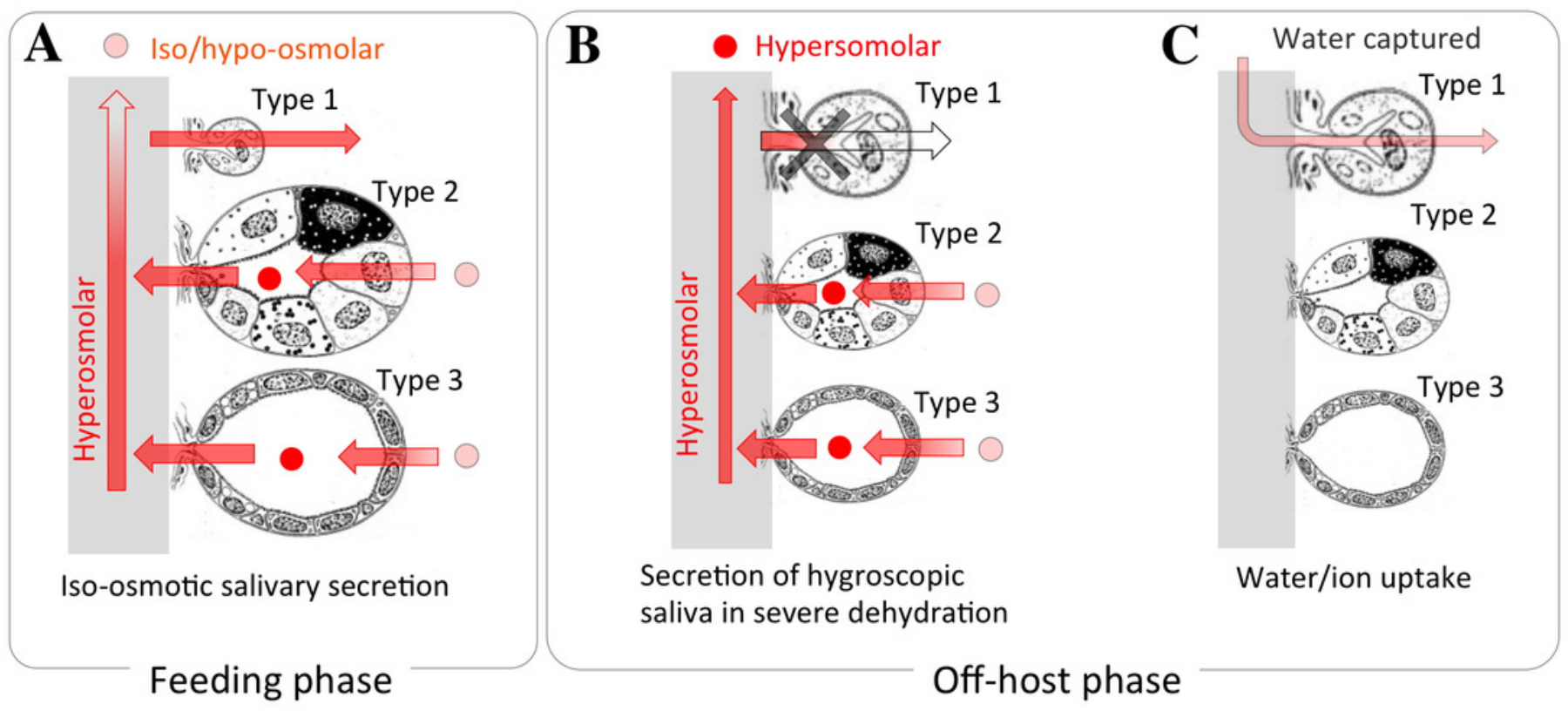

